\title{
Ultra-cold gases and the detection of the Earth's rotation: Bogoliubov space and gravitomagnetism
}

\author{
A. Camach中* \\ Departamento de Física, Universidad Autónoma Metropolitana-Iztapalapa \\ Apartado Postal 55-534, C.P. 09340, México, D.F., México. \\ E. Castellanos \\ ZARM, Universität Bremen, Am Fallturm, 28359 Bremen, Germany
}

(Dated: June 23, 2018)

\begin{abstract}
The present work analyzes the consequences of the gravitomagnetic effect of the Earth upon a bosonic gas in which the corresponding atoms have a non-vanishing orbital angular momentum. Concerning the ground state of the Bogoliubov space of this system we deduce the consequences, on the pressure and on the speed of sound, of the gravitomagnetic effect. We prove that the effect on a single atom is very small, but we also show that for some thermodynamical properties the consequences scale as a non-trivial function of the number of particles.
\end{abstract}

The deep analogies that can be found between electromagnetism and gravitation have a long history, a fact readily understood looking at Coulomb's law of electricity and Newton's law of gravitation [1]. The analogy was taken further and the possible existence of a magnetic component in the gravitational interaction between the Sun and the remaining celestial objects of our solar system was put forward in the nineteenth century [2, 3]. A more complete and profound relation between these two interactions emerged with the formulation of Einstein's theory of general relativity (GR), in which a gravitomagnetic field appears as an inexorable consequence of the presence of a current of mass-energy [1], though several theories of gravitation predict a gravitomagnetic contribution [4]. This effect has several consequences, one of them is related to the appearance of a gravitational Larmor theorem [5], i.e., the exterior gravity of a rotating source couples to the angular momentum of a test body and gives rise to a Larmor precession, in the same way as a magnetic field couples with the angular momentum of an electrically charged particle. Other manifestations of this effect are the so-called frame-dragging and geodetic precession [6]

The gravitomagnetic field is one of the most important predictions of GR and has no Newtonian counterpart. Currently the results associated to the motion of the LAGEOS and LAGEOS-II satellites provide observational evidence for this effect [7]. The extant spectrum of experiments, or of astrophysical observations, have a weight, mainly, in the classical realm [6]. Of course, there are also experimental proposals in the quantal world, namely, there is some evidence of a shift in the energy for some fermions $[8,9]$. The need for a more profound work in this direction is also a point that has to be underlined, an issue already addressed in the context of interferometry. either neutronic or atomic [10]. The quantal aspect of

\footnotetext{
* acq@xanum.uam.mx

$\dagger$ elias.castellanos@zarm.uni-bremen.de
}

the experimental corroboration has severe hurdles. Indeed, this fact is related to the smallness of this effect (the ratio between the gravitomagnetic and gravitoelectric effects of the Earth is $\sim 10^{-7}$ ). Clearly, the use of an atomic system in this context seems to be a very bad idea, due to the smallness of the gravitational passive mass of an atom. This last assertion is a correct one, though one must add that this statement is valid for one atomic system. The point here concerns the possibility of enhancing the consequences of this effect, upon a quantal system, resorting to a gas. The present work addresses this issue, namely, we show that in a Bose-Einstein condensate the effect upon a single atom appears, in the context of some thermodynamical properties as the speed of sound, multiplied by a non-trivial function of the number of particles. This fact acts as an enhancer for this effect.

Let us consider a rotating uncharged, idealized spherical body with mass $M$ and angular momentum $\vec{J}$. In the weak field and slow motion limit the gravitomagnetic field may be written, using the PPN parameters $\Delta_{1}$ and $\Delta_{2}$, as

$$
\vec{B}(\vec{x})=\left(\frac{7 \Delta_{1}+\Delta_{2}}{4}\right) \frac{G}{c^{2}} \frac{\vec{J}-3(\vec{J} \cdot \hat{x}) \hat{x}}{|\vec{x}|^{3}} .
$$

The case of GR implies $\frac{7 \Delta_{1}+\Delta_{2}}{4}=1[5]$, where $c$ is the speed of light, $\vec{x}$ is the position vector, $G$ is the Newtonian gravitational constant, and $\hat{x}$ is the unit vector related to $\vec{x}$. We now assume that this gravitomagnetic field couples to the orbital angular momentum of an atom in the same way as it does in the case of a classical angular momentum [5].

Following this analogy between gravitomagnetism and magnetism we may now write down the interaction Hamiltonian that describes the coupling between gravitomagnetism and the orbital angular momentum of an atom, here denoted by $\vec{L}$

$$
W=-\vec{L} \cdot \vec{B} .
$$


For the sake of simplicity we now impose some restrictions. Firstly, the Earth has a perfect spherical symmetry with a radius $R$, mass $M$, constant mass density, and rotation frequency equal to $\omega$. Under these conditions we have that [5]

$$
\vec{J}=\frac{2 M R^{2}}{5} \omega \vec{e}_{z}
$$

Clearly, the Hamiltonian for an atom must include this last term the one will be considered in the $N$-body Hamiltonian operator (assuming that the gas is so dilute that only the two-body interaction potential is required [11]). The system under study will be a Bose-Einstein gas enclosed in a container of volume $V$, particles of the gas are atoms with passive gravitational mass $m$ and located at a height $z<<R$ with respect to the Earth's surface. In addition, the interaction between two particles will be assumed to be dominated by $s$-scattering, i.e., the temperature of the system is very low $(k a<<1$, where $\vec{k}$ and $a$ are the wave vector and the scattering length, respectively) [12]. This entails the following Hamiltonian for the $N$-body system.

$$
\begin{gathered}
\hat{H}=\sum_{\vec{k}=0} \frac{\hbar^{2} k^{2}}{2 m} \hat{a}_{\vec{k}}^{\dagger} \hat{a}_{\vec{k}} \\
+\frac{U_{0}}{2 V} \sum_{\vec{k}=0} \sum_{\vec{p}=0} \sum_{\vec{q}=0} \hat{a}_{\vec{p}}^{\dagger} \hat{a}_{\vec{q}}^{\dagger} \hat{a}_{\vec{p}+\vec{k}} \hat{a}_{\vec{q}-\vec{k}} \\
+\sum_{\vec{k}=0} m g z \hat{a}_{\vec{k}}^{\dagger} \hat{a}_{\vec{k}}+\sum_{\vec{k}=0} \sum_{s=0, \pm 2} \frac{2 s g \omega R \hbar}{5 c^{2}} \hat{a}_{\vec{k}, s}^{\dagger} \hat{a}_{\vec{k}, s}, \\
U_{0}=\frac{4 \pi a \hbar^{2}}{m} .
\end{gathered}
$$

The last term in our Hamiltonian is related to the fact that the coupling between gravitomagnetism and orbital angular momentum is absent for the case of vanishing l. In addition, since we assume that the gas has a very low temperature (this phrase means smaller than the condensation temperature) then almost all the particles have $l=0$ and a few ones will have non-vanishing angular momentum and, at this point, we assume that they are in the $d$-state, i.e., $l=2$, because the symmetry requirements associated to the wave function discard the case of $l=1$ [11], i.e., the first case with non-vanishing angular momentum is $l=2$ and not $l=1$. The parameter $s$ denotes the five possibilities related to the eigenvalues of the operator $L_{z}$, namely $s= \pm 1,0, \pm 2$. These operators $\left(\hat{a}_{\vec{k}}\right.$ and $\left.\hat{a}_{\vec{k}}^{\dagger}\right)$ are bosonic creation and annihilation operators, and fulfill the usual Bose commutation relations. As an additional simplification, which does not restrict the validity of our results, we assume that all the particles in the $d$-state have eigenvalue for $L_{z}$ equal to $s=+2$. Very close to the temperature $T=0$, the second term in this Hamiltonian becomes [13]

$$
\begin{array}{r}
\sum_{\vec{k}=0} \sum_{\vec{p}=0} \sum_{\vec{q}=0} \hat{a}_{\vec{p}}^{\dagger} \hat{a}_{\vec{q}}^{\dagger} \hat{a}_{\vec{p}+\vec{k}} \hat{a}_{\vec{q}-\vec{k}}=N^{2}+2 N \sum_{\vec{k} \neq 0} \hat{a}_{\vec{k}}^{\dagger} \hat{a}_{\vec{k}} \\
+N \sum_{\vec{k} \neq 0}\left(\hat{a}_{\vec{k}}^{\dagger} \hat{a}_{-\vec{k}}^{\dagger}+\hat{a}_{\vec{k}} \hat{a}_{-\vec{k}}\right) .
\end{array}
$$

With these approximations the $N$-body Hamiltonian has the following structure

$$
\begin{array}{r}
\hat{H}=\frac{U_{0} N^{2}}{2 V}+m g z N+ \\
\sum_{\vec{k} \neq 0}\left[\frac{\hbar^{2} k^{2}}{2 m}+m g z+\frac{4 g \omega R \hbar}{5 c^{2}}+\frac{U_{0} N}{V}\right] \hat{a}_{\vec{k}}^{\dagger} \hat{a}_{\vec{k}} \\
+N \frac{U_{0}}{2 V}\left[\hat{a}_{\vec{k}}^{\dagger} \hat{a}_{-\vec{k}}^{\dagger}+\hat{a}_{\vec{k}} \hat{a}_{-\vec{k}}\right] .
\end{array}
$$

This Hamiltonian can be diagonalized introducing the Bogoliubov transformations [12]

$$
\begin{aligned}
& \hat{b}_{\vec{k}}=\frac{1}{\sqrt{1-\alpha_{k}^{2}}}\left[\hat{a}_{\vec{k}}+\alpha_{k} \hat{a}_{-\vec{k}}^{\dagger}\right], \\
& \hat{b}_{\vec{k}}^{\dagger}=\frac{1}{\sqrt{1-\alpha_{k}^{2}}}\left[\hat{a}_{\vec{k}}^{\dagger}+\alpha_{k} \hat{a}_{-\vec{k}}\right] .
\end{aligned}
$$

These two operators fulfill the same algebra related to $\hat{a}_{\vec{k}}$ and $\hat{a}_{\vec{k}}^{\dagger}$, i.e., they are also bosonic operators. In this last expression the following definitions have been introduced

$$
\begin{gathered}
\epsilon_{k}=\frac{\hbar^{2} k^{2}}{2 m}+m g z+\frac{4 g \omega R \hbar}{5 c^{2}}, \\
\alpha_{k}=1+\frac{V \epsilon_{k}}{U_{0} N}-\sqrt{\frac{V \epsilon_{k}}{U_{0} N}} \sqrt{2+\frac{V \epsilon_{k}}{U_{0} N}} .
\end{gathered}
$$

The final form for our Hamiltonian is

$$
\begin{array}{r}
\hat{H}=\frac{U_{0} N^{2}}{2 V}+m g z N \\
+\sum_{\vec{k} \neq 0}\left\{\sqrt{\epsilon_{k}\left(\epsilon_{k}+\frac{2 U_{0} N}{V}\right)} \hat{b}_{\vec{k}}^{\dagger} \hat{b}_{\vec{k}}\right. \\
\left.-\frac{1}{2}\left[\frac{U_{0} N}{V}+\epsilon_{k}-\sqrt{\epsilon_{k}\left(\epsilon_{k}+\frac{2 U_{0} N}{V}\right)}\right]\right\} .
\end{array}
$$


The last summation diverges, a result already known [14, 15], and this divergence disappears introducing the so-called pseudo-potential method, which implies that we must perform the following substitution [15]

$$
\begin{gathered}
-\frac{1}{2}\left[\frac{U_{0} N}{V}+\epsilon_{k}-\sqrt{\epsilon_{k}\left(\epsilon_{k}+\frac{2 U_{0} N}{V}\right)}\right] \\
-\frac{1}{2}\left[\frac{U_{0} N}{V}+\epsilon_{k}-\sqrt{\epsilon_{k}\left(\epsilon_{k}+\frac{2 U_{0} N}{V}\right)}-\frac{1}{2 \epsilon_{k}}\left(\frac{U_{0} N}{V}\right)^{2}\right] .
\end{gathered}
$$

Finally, this last summation will be approximated by an integral. It is noteworthy to mention that the original expression has as lower limit the condition $k \neq 0$, which implies that the integral does not have as lower limit the value 0 . In other words,

$$
\begin{aligned}
& -\frac{1}{2} \sum_{\vec{k} \neq 0}\left[\frac{U_{0} N}{V}+\epsilon_{k}-\sqrt{\epsilon_{k}\left(\epsilon_{k}+\frac{2 U_{0} N}{V}\right)}-\frac{1}{2 \epsilon_{k}}\left(\frac{U_{0} N}{V}\right)^{2}\right] \\
& =-\frac{\hbar^{2} V}{8 m \pi^{2}}\left(\frac{8 \pi a N}{V}\right)^{5 / 2} \int_{\alpha}^{\infty} f(x) d x
\end{aligned}
$$

In this last expression we have that

$$
\alpha^{2}=\left[1+\frac{4 \omega R \hbar}{5 m c^{2} z}\right] \frac{m g z V}{U_{0}} .
$$

Additionally

$$
\begin{gathered}
f(x)=x^{2}\left[1+x^{2}-x \sqrt{2+x^{2}}-\frac{1}{2 x^{2}}\right], \\
x=\sqrt{\frac{\epsilon_{k} V}{U_{0} N}} .
\end{gathered}
$$

With these conditions we deduce the final structure of the $N$-body Hamiltonian

$$
\hat{H}=E_{0}+\sum_{\vec{k} \neq 0} E_{k} \hat{b}_{\vec{k}}^{\dagger} \hat{b}_{\vec{k}} .
$$

In this last expression $E_{0}$ denotes the energy of the ground state of the corresponding Bogoliubov space [12].

$$
\begin{aligned}
E_{0}= & \frac{2 \pi a \hbar^{2} N^{2}}{m V}\left[1+\frac{128}{15} \sqrt{\frac{a^{3} N}{V \pi}}\right. \\
& \left.\left(1-\frac{15}{16 \sqrt{2}} \alpha\right)\right]+\alpha^{2} \frac{U_{0} N}{V} .
\end{aligned}
$$

On the other hand, we have that the energy of the Bogoliubov excitations $\left(E_{k}\right)$ is given by 12 ]

$$
E_{k}=\sqrt{\epsilon_{k}\left(\epsilon_{k}+\frac{2 U_{0} N}{V}\right)} .
$$

Concerning (18), if we impose the condition of vanishing gravitational constant, i.e. $g=0$, then we recover the usual Hamiltonian [15].

The pressure $\left(P_{0}=-\frac{\partial E_{0}}{\partial V}\right)$ and speed of sound $\left(v_{s}=\right.$ $\left.\sqrt{-\frac{V^{2}}{N m} \frac{\partial P_{0}}{\partial V}}\right)$ associated to the ground state of the Bogoliubov space become, respectively

$$
\begin{aligned}
P_{0}= & \frac{2 \pi a \hbar^{2} N^{2}}{m V^{2}}\left[1+\frac{194}{15} \sqrt{\frac{a^{3} N}{V \pi}}\right. \\
& \left.\left(1-\frac{15}{16 \sqrt{2}} \alpha\right)\right]+O\left(\alpha^{2}\right) \\
v_{s}^{2}= & \frac{4 \pi a \hbar^{2} N}{m^{2} V}\left[1+\frac{242}{15} \sqrt{\frac{a^{3} N}{V \pi}}\right. \\
& \left.\left(1-\frac{15}{16 \sqrt{2}} \alpha\right)\right]+O\left(\alpha^{2}\right)
\end{aligned}
$$

Notice that the possibility of detecting the term depending upon the gravitomagnetic effect $\left(\delta v_{s}^{g m}\right)$ requires that, if $\Delta v_{s}$ is the experimental error related to the measurement of the speed of sound, then $\Delta v_{s}<\left|\delta v_{s}^{g m}\right|$. In our case this entails

$$
\Delta\left(v_{s}\right)<\frac{3}{\sqrt{2}} \frac{a \omega g R}{c^{2}} N .
$$

The detection of the speed of sound in condensates has already a long history [16, 17]. The main difficulty, in the experimental context, is related to the fact that (for the case of an atom) $\frac{a \omega g R}{c^{2}} \sim 10^{-23}$. Nevertheless, this contribution to the speed of sound does not depend upon the density of particles but upon the number of particles. In other words, this tiny contribution is enhanced by the number of particles $(N)$ related to a bosonic gas. The density in condensation experiments ranges from $10^{13}$ to $10^{15}$ particles per cubic $\mathrm{cm}$ [18], hence for a condensate whose volume is $1.4 \mathrm{~cm}^{3}$ we have that

$$
\frac{3}{\sqrt{2}} \frac{a \omega g R}{c^{2}} N \sim 10^{-2} \mathrm{~m} / \mathrm{s} .
$$

Under these conditions an experimental uncertainty of $\Delta v_{s} \sim 10^{-3} \mathrm{~m} / \mathrm{s}$ would allow the detection of this field.

\section{ACKNOWLEDGMENTS}

This research was partially supported by DAAD grant A/09/77687 
[1] I. Ciufolini and J. A. Wheeler, Gravitation and Inertia (Princeton University Press, Princeton, New Jersey, 1995).

[2] G. Holzmüller, Z. Math. Phys. 15, 69-73 (1870).

[3] F. Tisserand, Comp. Rend. 75, 760-768 (1872).

[4] W.-T. Ni, Phys. Rev. D7, 2880-2883 (1973).

[5] B. Mashhoon, arXiv-gr-qc:0311030v2 (2008).

[6] I. Ciufolini, arXiv-gr-qc:0809:3219v1 (2008).

[7] I. Ciufolini, Class. Quantum Grav. 17, 2369-2380 (2000).

[8] B. J. Venema et al, Phys. Rev. Lett. 68, 135-138 (1992).

[9] B. Mashhoon, Phys. Lett. A198, 9-12 (1995).

[10] B. Mashhoon et al, Phys. Lett. A249, 161-166 (1998).

[11] R. K. Pathria, Statistical Mechanics (ButterworthHeinemann, Oxford, 1996).

[12] L. Pitaevski and S. Stringari, Bose-Einstein Condensation (Clarendon Press, Oxford, 2003).
[13] C. J. Pethick and H. Smith, Bose-Einstein Condensation in Dilute Gases (Cambridge University Press, Cambridge, 2004).

[14] G. F. Gribakin and V. V. Flambaum, Phys. Rev. A48, 546-563 (1993).

[15] M. Ueda, Fundamentals and New Frontiers of BoseEinstein Condensation (World Scientic, Singapore, 2010).

[16] M. R. Andrews et al, Phys. Rev. Lett. 79, 553-556 (1997).

[17] M. R. Andrews et al, Phys. Rev. Lett. 80, 2967-556 (1998).

[18] A. Griffin, T. Nikuni, and E. Zaremba, Bose-Condensed Gases at Finite Temperatures (Cambridge University Press, Cambridge, 2009). 